\title{
Some Mathematical Properties of Effective Resonator Model of 1D Dielectric Multilayer Structure
}

\author{
A. RudZIŃSKI* \\ Institute of Microelectronics and Optoelectronics \\ Warsaw University of Technology \\ Koszykowa 75, 00-665 Warszawa, Poland
}

(Received March 3, 2008)

\begin{abstract}
This paper presents a review of mathematical properties of effective resonator model of $1 \mathrm{D}$ dielectric multilayer structure. It constitutes a mathematical supplement to the previous papers, in which the model has been described.
\end{abstract}

PACS numbers: 42.25.Bs, 42.70.Qs

\section{Introduction}

The aim of this paper is to fill in the gaps left in the construction of the effective resonator model of $1 \mathrm{D}$ multilayer dielectric structure. In the previous papers [1-4] the model has been proposed and its physical background and interpretation $[1,2,5]$, as well as orthonormalization of modes [3, 4] have been described. However, there remain a few aspects of the model, which require a rigorous treatment or explanation. They are discussed in this paper, which is a mathematical supplement to the previous ones. Because of its purpose, general assumptions, notation and definitions of quantities (not recalled in this paper, except of a few cases) are the same as in [2-4].

Discussion of general properties of the model is the topic of Sect. 2. In the next three sections, conclusions are drawn for radiation (Sect. 3), substrate and guided modes (Sect. 4). The last section, Sect. 5, contains a summary of the paper.

\section{General properties of the model}

Property 1. Continuity conditions matrix $m_{j+1, j}$ in function of wave vector $\boldsymbol{k}$ obeys the relation $m_{j+1, j}\left(\boldsymbol{k}_{*}\right)=$ $m_{j+1, j}^{*}(\boldsymbol{k})$.

Proof. The explicit form of matrix $m_{j+1, j}$ is $[2,6]$ :

$$
\begin{aligned}
& m_{j+1, j}= \\
& \quad \frac{1}{2}\left(\begin{array}{ll}
K_{j}^{+} \exp \left(\mathrm{i} k_{z}^{(j)} L_{z}^{(j)}\right) & K_{j}^{-} \exp \left(-\mathrm{i} k_{z}^{(j)} L_{z}^{(j)}\right) \\
K_{j}^{-} \exp \left(\mathrm{i} k_{z}^{(j)} L_{z}^{(j)}\right) & K_{j}^{+} \exp \left(-\mathrm{i} k_{z}^{(j)} L_{z}^{(j)}\right)
\end{array}\right),
\end{aligned}
$$

with $K_{j}^{ \pm}$dependent on polarization

\footnotetext{
* e-mail: a.rudzinski@elka.pw.edu.pl
}

$$
K_{j}^{\mathrm{TE} \pm}=1 \pm \frac{k_{z}^{(j)}}{k_{z}^{(j+1)}}, K_{j}^{\mathrm{TM} \pm}=1 \pm \frac{n_{(j+1)}^{2}}{n_{(j)}^{2}} \frac{k_{z}^{(j)}}{k_{z}^{(j+1)}} .
$$

The behavior of matrix elements under the transformation $\boldsymbol{k} \rightarrow \boldsymbol{k}_{*}$ is to be examined. First, let $k_{z}^{(j)} \in \mathbb{R}$. The exponential terms in the matrix become their complex conjugates, because $k_{z}^{(j)}$ simply changes its sign. If $k_{z}^{(j+1)} \in \mathbb{R}$, it changes its sign as well and the coefficients $K_{j}^{ \pm} \in \mathbb{R}$ remain unchanged. If $k_{z}^{(j+1)} \notin \mathbb{R}$ then it is imaginary and does not change its sign, thus $K_{j}^{ \pm} \rightarrow K_{j}^{ \pm *}$. The second possibility is $k_{z}^{(j)} \notin \mathbb{R}$. In this case, the exponential terms are real and do not change. If $k_{z}^{(j+1)} \in \mathbb{R}$ then again $K_{j}^{ \pm} \rightarrow K_{j}^{ \pm *}$, while if $k_{z}^{(j+1)} \notin \mathbb{R}$ then $K_{j}^{ \pm} \in \mathbb{R}$ and these coefficients do not change. In all these cases $m_{j+1, j} \rightarrow m_{j+1, j}^{*}$.

Property 2. An immediate conclusion from property 1 is that under the transformation $\boldsymbol{k} \rightarrow \boldsymbol{k}_{*}: m_{j, i} \rightarrow m_{j, i}^{*}$, $w_{j, i} \rightarrow w_{j, i}^{*}, r_{\mathrm{L}} \rightarrow r_{\mathrm{L}}^{*}$ and $r_{\mathrm{R}} \rightarrow r_{\mathrm{R}}^{*}$. From this property of the reflection coefficients it also follows that $\rho_{\epsilon}\left(\boldsymbol{k}_{*}\right)=$ $\rho_{\epsilon}(\boldsymbol{k})$ and $\xi_{\epsilon}\left(\boldsymbol{k}_{*}\right)=\xi_{\epsilon}^{*}(\boldsymbol{k})$.

Property 3. Amplitudes of waves in two consecutive layers are bound by the matrix $m_{j+1, j}[2,6]$. Let $m_{j+1, j}^{\prime}$ be the matrix relating these amplitudes after a third layer with refractive index $n^{\prime}$ and width $L^{\prime}$ has been put between the $j$-th and $(j+1)$-th layer. In the limit $L^{\prime} \rightarrow 0$ :

$$
\lim _{L^{\prime} \rightarrow 0} m_{j+1, j}^{\prime}=m_{j+1, j}
$$

for any value of $n^{\prime}$. Thus, a placement of a layer with zero width is in fact a (useful) mathematical trick.

Property 4. Complex matrices $2 \times 2$ of form $\left(\begin{array}{cc}D^{*} & B \\ B^{*} & D\end{array}\right)$ and determinant equal to 1 , form, with the usual matrix multiplication, a group $\mathcal{T}_{2 \times 2}$. In particular, if $n_{(j)}=n_{(j+2)}=n_{(0)}$, then matrix $m_{j+2, j} \in \mathcal{T}_{2 \times 2}$.

Property 5. From properties 3 and 4 it follows that if $n_{(i)}=n_{(j)}=n_{(0)}$ and $j>i$, then matrix $m_{j, i} \in \mathcal{T}_{2 \times 2}$. 
Property 6. Coefficients $u_{j k \epsilon}$ and $v_{j k \epsilon}$ are defined by the expression $[2,3]$ :

$$
\begin{aligned}
& \left(\begin{array}{c}
u_{j k \epsilon} \\
v_{j k_{*} \epsilon}
\end{array}\right)=\left(\delta_{\epsilon \mathrm{TE}}+\frac{n_{(0)}}{n_{(j)}} \delta_{\epsilon \mathrm{TM}}\right) \\
& \quad \times\left\{\begin{array}{c}
m_{j, 0} \\
w_{j, 0}
\end{array}\right\}\left(\begin{array}{c}
1 \\
\xi_{\epsilon}^{*}(\boldsymbol{k})
\end{array}\right),
\end{aligned}
$$

where $\left\{\begin{array}{c}m_{j, i} \\ w_{j, i}\end{array}\right\}$ denotes $m_{j, i}$ if $j \geq i$, and $w_{j, i}$ if $j<i$. From this relation, one easily finds that $u_{i k \epsilon}, v_{i k_{*} \epsilon}$ and $u_{j k \epsilon}, v_{j k_{*} \epsilon}$ for $j>i$ are bound by the relation

$$
\left(\begin{array}{c}
u_{j k \epsilon} \\
v_{j k_{*} \epsilon}
\end{array}\right)=\left(\delta_{\epsilon \mathrm{TE}}+\frac{n_{(i)}}{n_{(j)}} \delta_{\epsilon \mathrm{TM}}\right) m_{j, i}\left(\begin{array}{c}
u_{i k \epsilon} \\
v_{i k_{*} \epsilon}
\end{array}\right) .
$$

Property 7. Properties 2 and 6 lead to $u_{j k_{*} \epsilon}=u_{j k \epsilon}^{*}$ and $v_{j k_{*} \epsilon}=v_{j k \epsilon}^{*}$.

Property 8. Electric field polarization versors [2] are defined as (in expressions (6)-(10) components $k_{x}$ and $k_{y}$ of vector $\boldsymbol{k}$ are real, but the component $k_{z}$ may be either real or imaginary):

$$
\boldsymbol{e}_{k \mathrm{TE}}=\frac{\boldsymbol{k} \times \boldsymbol{n}}{|\boldsymbol{k} \times \boldsymbol{n}|}, \quad \boldsymbol{e}_{k \mathrm{TM}}=\frac{\boldsymbol{e}_{k \mathrm{TE}} \times \boldsymbol{k}}{|\boldsymbol{k}|},
$$

where $\boldsymbol{n}$ is a versor parallel to the rotational symmetry axis of the structure, in the adopted coordinate system $\boldsymbol{n}=\boldsymbol{e}_{z}$ (although the choice of $-\boldsymbol{e}_{z}$ is equally good). It is easy to check that

$$
\boldsymbol{e}_{-k^{*} \epsilon}=-\gamma_{\epsilon} \boldsymbol{e}_{k \epsilon}^{*},
$$

where

$$
\gamma_{\epsilon}=\left\{\begin{array}{cc}
1, & \text { for } \epsilon=\mathrm{TE}, \\
-1, & \text { for } \epsilon=\mathrm{TM}
\end{array}\right.
$$

For $\boldsymbol{k} \| \boldsymbol{n}$, any versors perpendicular to $\boldsymbol{k}$ can be chosen for $\boldsymbol{e}_{k \mathrm{TE}}$ and $\boldsymbol{e}_{-k \mathrm{TE}}$, with the additional demand of satisfying (7), for consistence. Polarization versors of magnetic field are defined as:

$$
\boldsymbol{e}_{k \epsilon}^{H}=\frac{\boldsymbol{k} \times \boldsymbol{e}_{k \epsilon}}{|\boldsymbol{k}|} .
$$

For these versors

$$
\boldsymbol{e}_{-k^{*} \epsilon}^{H}=\gamma_{\epsilon} \boldsymbol{e}_{k \epsilon}^{H^{*}} \text {. }
$$

Property 9. Electric field distributions $\boldsymbol{\psi}_{k \epsilon}[3]$ obey $\boldsymbol{\psi}_{-k \epsilon}=-\gamma_{\epsilon} \boldsymbol{\psi}_{k \epsilon}^{*}$. Magnetic field distributions $\psi_{k \epsilon}^{H}$ can be found with the help of the formula

$$
\boldsymbol{\psi}_{k \epsilon}^{H}=\frac{\nabla \times \boldsymbol{\psi}_{k \epsilon}}{\mathrm{i} \mu_{0} \omega_{k}},
$$

and satisfy the relation $\boldsymbol{\psi}_{-k \epsilon}^{H}=\gamma_{\epsilon} \boldsymbol{\psi}_{k \epsilon}^{H *}$.

Property 10. Electric and magnetic field in the structure can be written as

$$
\begin{aligned}
& \boldsymbol{E}(\boldsymbol{r}, t)=\sum_{\epsilon} \int \mathrm{d}^{3} k \mathcal{E}_{k \epsilon} \boldsymbol{f}_{k \epsilon}(\boldsymbol{r}), \\
& \boldsymbol{H}(\boldsymbol{r}, t)=\sum_{\epsilon} \int \mathrm{d}^{3} k \mathcal{B}_{k \epsilon} \boldsymbol{f}_{k \epsilon}^{H}(\boldsymbol{r}),
\end{aligned}
$$

where $f_{k \epsilon}$ are the orthonormal modes, for which the orthonormality condition has the form [7]:

$$
\int \mathrm{d}^{3} r n^{2}(\boldsymbol{r}) \boldsymbol{f}_{q \lambda}^{*}(\boldsymbol{r}) \boldsymbol{f}_{k \epsilon}(\boldsymbol{r})=\delta_{\epsilon \lambda} \delta(\boldsymbol{k}-\boldsymbol{q}) .
$$

The integrations in (12) and (13) are to be conducted over wave vectors $\boldsymbol{k}$ for which $\boldsymbol{f}_{k \epsilon}$ form a linearly independent set.

The inner product of field distributions $\psi_{k \epsilon}$ is given by

$$
\begin{gathered}
\int \mathrm{d}^{3} r n^{2}(\boldsymbol{r}) \boldsymbol{\psi}_{q \lambda}^{*}(\boldsymbol{r}) \boldsymbol{\psi}_{k \epsilon}(\boldsymbol{r})=\rho_{\epsilon}^{2}(\boldsymbol{k}) \delta_{\epsilon \lambda} \\
\times\left[F_{k \epsilon} \delta(\boldsymbol{k}-\boldsymbol{q})+\tilde{F}_{k \epsilon} \delta\left(\boldsymbol{k}-\boldsymbol{q}_{*}\right)\right],
\end{gathered}
$$

where $F_{k \epsilon} \in \mathbb{R}_{+}$and $F_{k_{*} \epsilon}=F_{k \epsilon}, \tilde{F}_{k \epsilon} \in \mathbb{C}$ and $\tilde{F}_{k_{*} \epsilon}=\tilde{F}_{k \epsilon}^{*}$. Therefore, the relation between modes $\boldsymbol{\psi}_{k \epsilon}$ and orthonormal modes $\boldsymbol{f}_{k \epsilon}$ has to be of the form [3]:

$$
\boldsymbol{\psi}_{k \epsilon}(\boldsymbol{r})=r_{k \epsilon}\left[\boldsymbol{f}_{k \epsilon}(\boldsymbol{r})+s_{k \epsilon} \boldsymbol{f}_{k_{*} \epsilon}(\boldsymbol{r})\right] .
$$

It can be additionally demanded that $r_{k \epsilon} \in \mathbb{R}$ and $r_{k_{*} \epsilon}=r_{k \epsilon}$. It is easy to find, putting $\boldsymbol{\psi}_{k \epsilon}$ defined this way into (15), that

$$
s_{k \epsilon}= \begin{cases}\frac{F_{k \epsilon}-\sqrt{F_{k \epsilon}^{2}-\left|\tilde{F}_{k \epsilon}\right|^{2}}}{\tilde{F}_{k \epsilon}^{*}}, & \tilde{F}_{k \epsilon} \neq 0, \\ 0, & \tilde{F}_{k \epsilon}=0,\end{cases}
$$

and $s_{k_{*} \epsilon}=s_{k \epsilon}^{*}$. If $\left|s_{k \epsilon}\right| \neq 1$, then (16) can be simply inverted, giving

$$
\boldsymbol{f}_{k \epsilon}(\boldsymbol{r})=\frac{1}{r_{k \epsilon}} \frac{\boldsymbol{\psi}_{k \epsilon}(\boldsymbol{r})-s_{k \epsilon} \boldsymbol{\psi}_{k_{*} \epsilon}(\boldsymbol{r})}{1-\left|s_{k \epsilon}\right|^{2}},
$$

with

$$
r_{k \epsilon}=\rho_{\epsilon}(\boldsymbol{k}) \sqrt{\frac{F_{k \epsilon}}{1+\left|s_{k \epsilon}\right|^{2}}}, \quad \text { for } \quad\left|s_{k \epsilon}\right| \neq 1 .
$$

Property 11. It is obvious that if $F_{k \epsilon}=\left|\tilde{F}_{k \epsilon}\right|$ then $\left|s_{k \epsilon}\right|=1$. In this case, from (16),

$$
\begin{aligned}
& \left|s_{k \epsilon}\right|=1 \Rightarrow \\
& s_{k \epsilon} \boldsymbol{\psi}_{k_{*} \epsilon}=r_{k \epsilon}\left(s_{k \epsilon} \boldsymbol{f}_{k_{*} \epsilon}+\boldsymbol{f}_{k \epsilon}\right)=\boldsymbol{\psi}_{k \epsilon},
\end{aligned}
$$

meaning that $\boldsymbol{\psi}_{k \epsilon}$ and $\boldsymbol{\psi}_{k_{*} \epsilon}$ describe the same mode. Because, in general, $\boldsymbol{f}_{k \epsilon}$ can be related only to $\boldsymbol{\psi}_{k \epsilon}$ or $\boldsymbol{\psi}_{k_{*} \epsilon}$, which for $\left|s_{k \epsilon}\right|=1$ represent the same mode, then, in this case, $\boldsymbol{f}_{k \epsilon}$ has to be proportional to $\boldsymbol{\psi}_{k \epsilon}$. This implies that

$$
\left|s_{k \epsilon}\right|=1 \Rightarrow s_{k \epsilon} \boldsymbol{f}_{k_{*} \epsilon}=\boldsymbol{f}_{k \epsilon}
$$

and (16) becomes simply $\boldsymbol{\psi}_{k \epsilon}=2 r_{k \epsilon} \boldsymbol{f}_{k \epsilon}$. However, because of (21), the set of wave vectors, over which the integrations in (12) and (13) are performed, must not contain both $\boldsymbol{k}$ and $\boldsymbol{k}_{*}$ if $\left|s_{k \epsilon}\right|=1$. It can be chosen that wave vectors $\boldsymbol{k}$, for which $\left|s_{k \epsilon}\right|=1$ and $k_{z}<0$, are discarded. With this choice, the second delta function in (15) is always 0 (except for $k_{z}=0$, when it duplicates the first one, but a delta function on the edge of the integration interval introduces a $\frac{1}{2}$ factor) and $r_{k \epsilon}$ and the relation between $\boldsymbol{f}_{k \epsilon}$ and $\boldsymbol{\psi}_{k \epsilon}$ become

$$
\left|s_{k \epsilon}\right|=1 \Rightarrow\left\{\begin{array}{c}
r_{k \epsilon}=\rho_{\epsilon}(\boldsymbol{k}) \frac{\sqrt{F_{k \epsilon}}}{2} \\
\boldsymbol{f}_{k \epsilon}(\boldsymbol{r})=\frac{\boldsymbol{\psi}_{k \epsilon}(\boldsymbol{r})}{\rho_{\epsilon}(\boldsymbol{k}) \sqrt{F_{k \epsilon}}} .
\end{array}\right.
$$

Property 12. It is easy to check that $\boldsymbol{f}_{-k \epsilon}=-\gamma_{\epsilon} \boldsymbol{f}_{k \epsilon}^{*}$. Magnetic field distributions $\boldsymbol{f}_{k \epsilon}^{H}$, related to electric field 
distributions $\boldsymbol{f}_{k \epsilon}$, are given by

$$
\boldsymbol{f}_{k \epsilon}^{H}=\frac{\nabla \times f_{k \epsilon}}{\mathrm{i} \mu_{0} \omega_{k}}
$$

and satisfy the relation $\boldsymbol{f}_{-k \epsilon}^{H}=\gamma_{\epsilon} \boldsymbol{f}_{k \epsilon}^{H^{*}}$. Therefore, if electric and magnetic field given by (12) and (13) are real, then $\mathcal{E}_{k \epsilon}^{*}=-\gamma_{\epsilon} \mathcal{E}_{-k \epsilon}$ and $\mathcal{B}_{k \epsilon}^{*}=\gamma_{\epsilon} \mathcal{B}_{-k \epsilon}$.

Property 13. The relation (20) leads to the conclusion that

$$
\begin{aligned}
\left|s_{k \epsilon}\right|= & \Rightarrow \\
s_{k \epsilon} & = \begin{cases}u_{j k \epsilon} / v_{j k \epsilon}, & \text { if } k_{z}^{(j)} \in \mathbb{R}, \\
u_{j k \epsilon} / u_{j k \epsilon}^{*}=v_{j k \epsilon}^{*} / v_{j k \epsilon}, & \text { otherwise. }\end{cases}
\end{aligned}
$$

In particular, for the layer $j=0$, one obtains

$$
\left|s_{k \epsilon}\right|=1 \Rightarrow s_{k \epsilon}=\xi_{\epsilon}^{*}(\boldsymbol{k}) .
$$

From (20), (11), (21) and (23) one also finds that

$$
\left|s_{k \epsilon}\right|=1 \Rightarrow\left\{\begin{array}{c}
s_{k \epsilon} \boldsymbol{\psi}_{k_{*} \epsilon}^{H}=\boldsymbol{\psi}_{k \epsilon}^{H}, \\
s_{k \epsilon} \boldsymbol{f}_{k_{*} \epsilon}^{H}=\boldsymbol{f}_{k \epsilon}^{H} .
\end{array}\right.
$$

Property 14. If $\left|s_{k \epsilon}\right|=1$ then $\boldsymbol{f}_{k \epsilon}$ and $\boldsymbol{f}_{k_{*} \epsilon}$ describe the same mode, which implies that $\mathcal{E}_{k \epsilon} \boldsymbol{f}_{k \epsilon}=\mathcal{E}_{k_{*} \epsilon} \boldsymbol{f}_{k_{*} \epsilon}$. Obviously, the same condition holds for magnetic field: $\mathcal{B}_{k \epsilon} \boldsymbol{f}_{k \epsilon}^{H}=\mathcal{B}_{k_{*} \epsilon} \boldsymbol{f}_{k_{*} \epsilon}^{H}$. With (21) and (26), these conditions constitute the following constraints for the amplitudes of modes:

$$
\left|s_{k \epsilon}\right|=1 \Rightarrow\left\{\begin{array}{l}
\mathcal{E}_{k_{*} \epsilon}=s_{k \epsilon} \mathcal{E}_{k \epsilon}, \\
\mathcal{B}_{k_{*} \epsilon}=s_{k \epsilon} \mathcal{B}_{k \epsilon} .
\end{array}\right.
$$

Property 15. $s_{k \epsilon}$ is a solution of the equation

$$
2 F_{k \epsilon} s_{k \epsilon}=\tilde{F}_{k \epsilon}\left(1+\left|s_{k \epsilon}\right|^{2}\right) \text {, }
$$

which, for $\tilde{F}_{k \epsilon} \neq 0$, has two solutions

$$
s_{k \epsilon}^{ \pm}=\frac{F_{k \epsilon} \pm \sqrt{F_{k \epsilon}^{2}-\left|\tilde{F}_{k \epsilon}\right|^{2}}}{\tilde{F}_{k \epsilon}^{*}},
$$

accompanied by $r_{k \epsilon}^{+}$or $r_{k \epsilon}^{-}$, respectively. It is very easy to check that $s_{k \epsilon}^{+}=1 / s_{k \epsilon}^{-*}$ and $r_{k \epsilon}^{+}=\left|s_{k \epsilon}^{-}\right| r_{k \epsilon}^{-}$. Therefore, if $\left|s_{k \epsilon}^{ \pm}\right| \neq 1$, using $s_{k \epsilon}^{+}$and $s_{k \epsilon}^{-}$in (18) one obtains $\boldsymbol{f}_{k \epsilon}^{+}$and $\boldsymbol{f}_{k_{*} \epsilon}^{-}$, which are related by

$$
\boldsymbol{f}_{k \epsilon}^{+}(\boldsymbol{r})=\frac{s_{k \epsilon}^{-}}{\left|s_{k \epsilon}^{-}\right|} \boldsymbol{f}_{k_{*} \epsilon}^{-}(\boldsymbol{r}) \text {. }
$$

If $\left|s_{k \epsilon}^{ \pm}\right|=1$, then $s_{k \epsilon}^{+}=s_{k \epsilon}^{-}$. Thus, one obtains all the modes of the structure using only $s_{k \epsilon}^{-}$(as it has been chosen in (17)) or only $s_{k \epsilon}^{+}$.

\section{Properties of radiation modes}

Property 16. For any radiation mode $\left|\xi_{\epsilon}(\boldsymbol{k})\right|<1$.

Proof. For a radiation mode $\left|r_{\mathrm{L}}\right|<1$ and $\left|r_{\mathrm{R}}\right|<1$. Therefore, using the triangle inequality

$$
\begin{aligned}
& \left|\xi_{\epsilon}(\boldsymbol{k})\right| \leq \frac{\left|r_{\mathrm{R}}\right|\left(1-\left|r_{\mathrm{L}}\right|^{2}\right)+\left|r_{\mathrm{L}}\right|\left(1-\left|r_{\mathrm{R}}\right|^{2}\right)}{1-\left|r_{\mathrm{L}} r_{\mathrm{R}}\right|^{2}} \\
& \quad=\frac{\left|r_{\mathrm{R}}\right|+\left|r_{\mathrm{L}}\right|}{1+\left|r_{\mathrm{L}} r_{\mathrm{R}}\right|}<1 .
\end{aligned}
$$

Property 17. For any radiation mode $F_{k \epsilon}>\left|\tilde{F}_{k \epsilon}\right|$.
Proof. For a radiation mode

$$
\begin{aligned}
& F_{k \epsilon}=4 \pi^{3} n_{(0)}^{2}\left[\left|k_{z}^{(\mathrm{R})}\right|\left(\left|u_{\mathrm{R} k \epsilon}\right|^{2}+\left|v_{\mathrm{R} k \epsilon}\right|^{2}\right)\right. \\
& \left.+\left|k_{z}^{(\mathrm{L})}\right|\left(\left|u_{\mathrm{L} k \epsilon}\right|^{2}+\left|v_{\mathrm{L} k \epsilon}\right|^{2}\right)\right] /\left|k_{z}\right|, \\
& \tilde{F}_{k \epsilon}=8 \pi^{3} n_{(0)}^{2} \frac{\left|k_{z}^{(\mathrm{R})}\right| u_{\mathrm{R} k \epsilon} v_{\mathrm{R} k \epsilon}^{*}+\left|k_{z}^{(\mathrm{L})}\right| u_{\mathrm{L} k \epsilon} v_{\mathrm{L} k \epsilon}^{*}}{\left|k_{z}\right|} .
\end{aligned}
$$

Using the triangle inequality, one finds that

$$
\begin{aligned}
& \left|\tilde{F}_{k \epsilon}\right| \leq 8 \pi^{3} n_{(0)}^{2} \\
& \quad \times \frac{\left|k_{z}^{(\mathrm{R})}\right|\left|u_{\mathrm{R} k \epsilon}\right|\left|v_{\mathrm{R} k \epsilon}\right|+\left|k_{z}^{(\mathrm{L})}\right|\left|u_{\mathrm{L} k \epsilon}\right|\left|v_{\mathrm{L} k \epsilon}\right|}{\left|k_{z}\right|},
\end{aligned}
$$

thus

$$
\begin{aligned}
& F_{k \epsilon}-\left|\tilde{F}_{k \epsilon}\right| \geq 4 \pi^{3} n_{(0)}^{2} \\
& \quad \times \frac{\left|k_{z}^{(\mathrm{R})}\right|\left(\left|u_{\mathrm{R} k \epsilon}\right|-\left|v_{\mathrm{R} k \epsilon}\right|\right)^{2}+\left|k_{z}^{(\mathrm{L})}\right|\left(\left|u_{\mathrm{L} k \epsilon}\right|-\left|v_{\mathrm{L} k \epsilon}\right|\right)^{2}}{\left|k_{z}\right|} \\
& \quad \geq 0 .
\end{aligned}
$$

However, if $F_{k \epsilon}=\left|\tilde{F}_{k \epsilon}\right|$, then $\left|s_{k \epsilon}\right|=1$ and $s_{k \epsilon}=\xi_{\epsilon}^{*}(\boldsymbol{k})$ (property 13), but for a radiation mode it stands in contradiction to property (16). Hence, for radiation modes $F_{k \epsilon} \neq\left|\tilde{F}_{k \epsilon}\right|$ and finally $F_{k \epsilon}>\left|\tilde{F}_{k \epsilon}\right|$.

Property 18. Property 17 leads to the conclusion that for radiation modes $\left|s_{k \epsilon}\right|<1$, thus, radiation modes $\boldsymbol{f}_{k \epsilon}$ form an orthonormal set. From (16) it follows that field distributions $\boldsymbol{\psi}_{k \epsilon}$ of radiation modes form a linearly independent set.

\section{Properties of substrate and guided modes}

Property 19. Let $n_{(\mathrm{R})}<n_{(0)}$ and let the angle of incidence $\vartheta$ be greater than the total reflection angle $\vartheta_{\mathrm{T}}=\arcsin \left(n_{(\mathrm{R})} / n_{(0)}\right)$. Under these conditions $\xi_{\epsilon}^{*}(\boldsymbol{k})=r_{\mathrm{R}} \exp \left(2 \mathrm{i} k_{z} L_{z}\right)$ and relation (4) with $j=N_{\mathrm{R}}$ describes reflection and transmission of a plane wave through a stack of dielectric layers [4]. For this reason, $v_{\mathrm{R} k \epsilon}=0$.

If $n_{(\mathrm{L})}<n_{(0)}$ and $\vartheta>\vartheta_{\mathrm{T}}=\arcsin \left(n_{(\mathrm{L})} / n_{(0)}\right)$ then $v_{\mathrm{Lk \epsilon}}=0$ for the same reason.

Property 20. If $v_{\mathrm{L} k \epsilon}=0$ or $v_{\mathrm{R} k \epsilon}=0$, then $\forall_{j} k_{z}^{(j)} \in$ $\mathbb{R} \Rightarrow\left|v_{j k \epsilon}\right|=\left|u_{j k \epsilon}\right|$.

Proof. If $v_{\mathrm{Lk \epsilon}}=0$, Eq. (5) and property 7 give

$$
\begin{aligned}
& \left(\begin{array}{c}
u_{j k \epsilon} \\
v_{j k \epsilon}^{*}
\end{array}\right)=\left(\delta_{\epsilon \mathrm{TE}}+\frac{n_{(\mathrm{L})}}{n_{(j)}} \delta_{\epsilon \mathrm{TM}}\right) \\
& \times\left(\begin{array}{c}
\left(m_{j, \mathrm{~L}}\right)_{11} \\
\left(m_{j, \mathrm{~L}}\right)_{21}
\end{array}\right) u_{\mathrm{L} k \epsilon} .
\end{aligned}
$$

This means that

$$
\frac{v_{j k \epsilon}^{*}}{u_{j k \epsilon}}=\frac{\left(m_{j, \mathrm{~L}}\right)_{21}}{\left(m_{j, \mathrm{~L}}\right)_{11}} .
$$

Let $j>2-N_{\mathrm{L}}$ and widths $L^{(j-1)}=L^{\left(1-N_{\mathrm{L}}\right)}=0$. Property 3 states that in this case $\left(1-N_{\mathrm{L}}\right)$-th and $(j-1)$-th layer have no physical meaning, therefore their refractive 
indices can be chosen arbitrarily without any impact on generality. Let $n_{(\mathrm{L}+1)}=n_{(j-1)}=n_{(0)}$. In this situation, $m_{j-1, L+1} \in \mathcal{T}_{2 \times 2}$ (property 5). Therefore

$$
\begin{aligned}
m_{j, \mathrm{~L}} & =m_{j, j-1} m_{j-1, \mathrm{~L}+1} m_{\mathrm{L}+1, \mathrm{~L}} \\
& =m_{j, j-1}\left(\begin{array}{cc}
D^{*} & B \\
B^{*} & D
\end{array}\right) m_{\mathrm{L}+1, \mathrm{~L}} \\
& =\frac{1}{4}\left(\begin{array}{ll}
K_{j-1}^{+} & K_{j-1}^{-} \\
K_{j-1}^{-} & K_{j-1}^{+}
\end{array}\right)\left(\begin{array}{ll}
D^{*} & B \\
B^{*} & D
\end{array}\right) \\
& \times\left(\begin{array}{ll}
K_{\mathrm{L}}^{+} & K_{\mathrm{L}}^{-} \\
K_{\mathrm{L}}^{-} & K_{\mathrm{L}}^{+}
\end{array}\right)
\end{aligned}
$$

and

$$
\begin{aligned}
& \left(m_{j, \mathrm{~L}}\right)_{11}=B K_{j-1}^{+} K_{\mathrm{L}}^{-}+B^{*} K_{j-1}^{-} K_{\mathrm{L}}^{+} \\
& \quad+D K_{j-1}^{-} K_{\mathrm{L}}^{-}+D^{*} K_{j-1}^{+} K_{\mathrm{L}}^{+}, \\
& \left(m_{j, \mathrm{~L}}\right)_{21}=B K_{j-1}^{-} K_{\mathrm{L}}^{-}+B^{*} K_{j-1}^{+} K_{\mathrm{L}}^{+} \\
& \quad+D K_{j-1}^{+} K_{\mathrm{L}}^{-}+D^{*} K_{j-1}^{-} K_{\mathrm{L}}^{+} .
\end{aligned}
$$

Under established assumptions $K_{\mathrm{L}}^{+}=\left(K_{\mathrm{L}}^{-}\right)^{*}$. If $k_{z}^{(j)} \in \mathbb{R}$ then both $K_{j-1}^{ \pm} \in \mathbb{R}$ and $\left(m_{j, \mathrm{~L}}\right)_{11}=\left(m_{j, L}\right)_{21}^{*}$, hence $\left|v_{j k \epsilon}\right|=\left|u_{j k \epsilon}\right|$. The situation in which $v_{\mathrm{R} k \epsilon}=0$ can be obtained from the previous one with transformation $\boldsymbol{k} \rightarrow \boldsymbol{k}_{*}$, which, with property 7 , leads immediately to the same conclusion.

It can be assumed without loss of generality that $n_{(\mathrm{R})}<n_{(\mathrm{L})}$ (the case with the opposite relation is equivalent to the assumed one after the transformation $\left.\boldsymbol{k} \rightarrow \boldsymbol{k}_{*}\right)$. With this assumption, $k_{z}^{(\mathrm{L})} \in \mathbb{R}$.

Property 21. For a substrate mode [4]:

$$
\begin{aligned}
& F_{k \epsilon}=4 \pi^{3} n_{(0)}^{2}\left|\frac{k_{z}^{(\mathrm{L})}}{k_{z}}\right|\left(\left|u_{\mathrm{L} k \epsilon}\right|^{2}+\left|v_{\mathrm{L} k \epsilon}\right|^{2}\right), \\
& \tilde{F}_{k \epsilon}=8 \pi^{3} n_{(0)}^{2}\left|\frac{k_{z}^{(L)}}{k_{z}}\right| u_{\mathrm{L} k \epsilon} v_{\mathrm{L} k \epsilon}^{*} .
\end{aligned}
$$

Because of property 20, for substrate modes $\left|\tilde{F}_{k \epsilon}\right|=F_{k \epsilon}$. Thus, substrate modes are subject to properties and constraints of modes with $\left|s_{k \epsilon}\right|=1$.

Property 22. For guided modes

$$
\begin{aligned}
& F_{k \epsilon}= \\
& \begin{cases}4 \pi^{2} \int_{-\infty}^{\infty} \mathrm{d} z n^{2} z\left|\phi_{k \epsilon}(z)\right|^{2}, & \text { if } \rho_{\epsilon}(\boldsymbol{k}) \neq 0, \\
0, & \text { if } \rho_{\epsilon}(\boldsymbol{k})=0,\end{cases} \\
& \tilde{F}_{k \epsilon}= \\
& \begin{cases}4 \pi^{2} \int_{-\infty}^{\infty} \mathrm{d} z n^{2} z \boldsymbol{\phi}_{k_{*} \epsilon}^{*}(z) \phi_{k \epsilon}(z), & \text { if } \rho_{\epsilon}(\boldsymbol{k}) \neq 0, \\
0, & \text { if } \rho_{\epsilon}(\boldsymbol{k})=0,\end{cases}
\end{aligned}
$$

where

$$
\begin{aligned}
& \int_{-\infty}^{\infty} \mathrm{d} z n^{2}(z)\left|\phi_{k \epsilon}(z)\right|^{2}=\frac{n_{(\mathrm{L})}^{2}\left|u_{\mathrm{L} k \epsilon}\right|^{2}}{2\left|k_{z}^{(\mathrm{L})}\right|}+\frac{n_{(\mathrm{R})}^{2}\left|u_{\mathrm{R} k \epsilon}\right|^{2}}{2\left|k_{z}^{(\mathrm{R})}\right|} \\
& \quad+\sum_{-N_{\mathrm{L}}<j<N_{\mathrm{R}}} n_{(j)}^{2} L_{z}^{(j)}\left\{\left[\left|u_{j k \epsilon}\right|^{2} \exp \left(-\operatorname{Im}\left(k_{z}^{(j)}\right) L_{z}^{(j)}\right)\right.\right.
\end{aligned}
$$

$$
\begin{aligned}
& \left.+\left|v_{j k \epsilon}\right|^{2} \exp \left(\operatorname{Im}\left(k_{z}^{(j)}\right) L_{z}^{(j)}\right)\right] \frac{\sinh \left(\operatorname{Im}\left(k_{z}^{(j)}\right) L_{z}^{(j)}\right)}{\operatorname{Im}\left(k_{z}^{(j)}\right) L_{z}^{(j)}} \\
& +2 \operatorname{Re}\left(u_{j k \epsilon} v_{j k \epsilon} e_{k_{*}^{*} \epsilon_{\epsilon}}^{*} \boldsymbol{e}_{k^{(j)} \epsilon} \exp \left(\mathrm{i} \operatorname{Re}\left(k_{z}^{(j)}\right) L_{z}^{(j)}\right)\right) \\
& \left.\times \frac{\sin \left(\operatorname{Re}\left(k_{z}^{(j)}\right) L_{z}^{(j)}\right)}{\operatorname{Re}\left(k_{z}^{(j)}\right) L_{z}^{(j)}}\right\}
\end{aligned}
$$

and

$$
\begin{gathered}
\int_{-\infty}^{\infty} \mathrm{d} z n^{2}(z) \phi_{k_{*} \epsilon}^{*}(z) \phi_{k \epsilon}(z)=\frac{n_{(\mathrm{L})}^{2} u_{\mathrm{L} k \epsilon}^{2}}{2\left|k_{z}^{(\mathrm{L})}\right|}+\frac{n_{(\mathrm{R})}^{2} u_{\mathrm{R} k \epsilon}^{2}}{2\left|k_{z}^{(\mathrm{R})}\right|} \\
+\sum_{-N_{\mathrm{L}}<j<N_{\mathrm{R}}} n_{(j)}^{2} L_{z}^{(j)}\left\{\left[u_{j k \epsilon}^{2} \exp \left(\mathrm{i} k_{z}^{(j)} L_{z}^{(j)}\right)\right.\right. \\
\left.+v_{j k \epsilon}^{* 2} \exp \left(-\mathrm{i} k_{z}^{(j)} L_{z}^{(j)}\right)\right] \boldsymbol{e}_{k_{*}^{*} \epsilon_{\epsilon} \epsilon_{k}}^{\boldsymbol{e}_{k^{(j)}}} \frac{\sin \left(k_{z}^{(j)} L_{z}^{(j)}\right)}{k_{z}^{(j)} L_{z}^{(j)}} \\
\left.+2 u_{j k \epsilon} v_{j k \epsilon}^{*} \boldsymbol{e}_{k^{(j) *} \epsilon}^{*} \boldsymbol{e}_{k^{(j)} \epsilon}\right\} .
\end{gathered}
$$

Applying the properties inherent to modes, for which $\left|s_{k \epsilon}\right|=1$, one arrives consistently at $F_{k \epsilon}=\left|\tilde{F}_{k \epsilon}\right|$, thus, both of these equalities indeed occur for guided modes.

\section{Summary}

In this paper, various mathematical properties of the effective resonator model of 1D dielectric multilayer structure have been discussed. In particular, effects of transformation $\boldsymbol{k} \rightarrow \boldsymbol{k}_{*}$ have been described. It has been pointed out that usage of a "virtual" layer with zero width can be a convenient mathematical trick. A few important relations between modes of the structure have been presented. Special attention has been paid to properties of modes for which $\left|s_{k \epsilon}\right|=1$ and it has been shown that they apply to substrate and guided modes. The conclusions from the discussion in this paper are essential for a correct usage of the effective resonator model in calculations.

\section{References}

[1] A. Rudziński, A. Tyszka-Zawadzka, P. Szczepański, Proc. SPIE 5950, 59501A (2005).

[2] A. Rudziński, Acta Phys. Pol. A 111, 323 (2007).

[3] A. Rudziński, Acta Phys. Pol. A 112, 495 (2007); erratum: Acta Phys. Pol. A 112, 1327 (2007).

[4] A. Rudziński, Acta Phys. Pol. A 112, 505 (2007); erratum: Acta Phys. Pol. A 113, 1709 (2008).

[5] A. Rudziński, A. Tyszka-Zawadzka, P. Szczepański, Opt. Quant. Electron. 39, 501 (2007).

[6] P. Yeh, A. Yariv, C.-S. Hong, J. Opt. Soc. Am. 67, 423 (1977).

[7] R.J. Glauber, M. Lewenstein, Phys. Rev. A 43, 467 (1991). 